\title{
NMR and Mössbauer study of spin dynamics and electronic structure of $\mathrm{Fe}_{2+x} \mathrm{~V}_{1-x} \mathrm{Al}$ and $\mathrm{Fe}_{2} \mathrm{VGa}$
}

\author{
C. S. Lue, Yang Li, and Joseph H. Ross, Jr. \\ Department of Physics, Texas A\&M University, College Station, Texas 77843-4242, USA \\ George M. Irwin \\ Department of Chemistry and Physics, Lamar University, Beaumont, Texas 77710, USA
}

(Received 19 November 2002; revised manuscript received 22 April 2003; published 19 June 2003)

\begin{abstract}
In order to assess the magnetic ordering process in $\mathrm{Fe}_{2} \mathrm{VAl}$ and the related material $\mathrm{Fe}_{2} \mathrm{VGa}$, we have carried out nuclear magnetic resonance (NMR) and Mössbauer studies. ${ }^{27} \mathrm{Al}$ NMR relaxation measurements covered the temperature range $4-500 \mathrm{~K}$ in $\mathrm{Fe}_{2+x} \mathrm{~V}_{1-x} \mathrm{Al}$ samples. We found a peak in the NMR spin-lattice relaxation rate, ${ }^{27}\left(T_{1}^{-1}\right)$, corresponding to the magnetic transitions in each of these samples. These peaks appear at 125 $\mathrm{K}, 17 \mathrm{~K}$, and $165 \mathrm{~K}$ for $x=0.10,0$, and -0.05 , respectively, and we connect these features with critical slowing down of the localized antisite defects. Mössbauer measurements for $\mathrm{Fe}_{2} \mathrm{VAl}$ and $\mathrm{Fe}_{2} \mathrm{VGa}$ showed lines with no hyperfine splitting, and isomer shifts nearly identical to those of the corresponding sites in $\mathrm{Fe}_{3} \mathrm{Al}$ and $\mathrm{Fe}_{3} \mathrm{Ga}_{\text {, }}$ respectively. We show that a model in which local band filling leads to magnetic regions in the samples, in addition to the localized antisite defects, can account for the observed magnetic ordering behavior.
\end{abstract}

DOI: 10.1103/PhysRevB.67.224425

PACS number(s): 75.20.En, 76.60.Jx, 76.80.+y, 71.20.Lp

\section{INTRODUCTION}

In recent years, much attention has been focused on inhomogeneous magnetic materials and on their implications in both technological applications and fundamental physics. Granular ferromagnets have been widely investigated since the discovery of giant magnetoresistance (GMR) in these alloys. ${ }^{1,2}$ GMR in granular alloys is associated with the spindependent scattering of conduction electrons by disordered magnetic clusters, while in ordered compounds GMR may be associated with scattering from thermal disorder near $T_{c} \cdot{ }^{3}$ The Heusler alloys $\mathrm{Fe}_{2+x} \mathrm{~V}_{1-x} \mathrm{Al}$ and $\mathrm{Fe}_{2+x} \mathrm{~V}_{1-x} \mathrm{Ga}$ are ordered intermetallics which exhibit GMR near $T_{c},{ }^{4,5}$ although in addition to thermal disorder, localized magnetic clusters play a prominent role in these materials, even for the nominally nonmagnetic composition $x=0 .{ }^{6,7}$ There has been considerable interest in understanding the role of these clusters for the magnetic transitions, and the unusual electronic behavior associated with these materials. ${ }^{8-10}$

Both $\mathrm{Fe}_{2} \mathrm{VAl}$ and $\mathrm{Fe}_{2} \mathrm{VGa}$ have been shown to be semimetallic and intrinsically nonmagnetic, via electronic structure calculations ${ }^{11-15}$ NMR measurements, ${ }^{7,16,17}$ and optical measurements. ${ }^{18,19}$ These materials adopt the Heusler structure ( $\mathrm{L}_{1}$, or cF16 \#225, $\mathrm{AlCu}_{2} \mathrm{Mn}$-type), having a bccbased lattice in which each Fe ( $8 c$ position) has four $\mathrm{V}$ and four $\mathrm{Al}$ or $\mathrm{Ga}$ near-neighbors. Complete substitution by $\mathrm{Fe}$ on the $\mathrm{V}$ sites ( $4 b$ positions) produces the $\mathrm{Fe}_{3} \mathrm{Al}$ structure, $\mathrm{DO}_{3} \cdot \mathrm{Fe}_{3} \mathrm{Al}$ is a metallic ferromagnet in which both Fe sites carry a magnetic moment. ${ }^{20} \mathrm{In}_{\mathrm{Fe}_{2}+x} \mathrm{~V}_{1-x} \mathrm{Al} T_{c}$ goes monotonically to zero as $x$ goes to zero, ${ }^{9}$ although $\mathrm{Fe}_{2} \mathrm{VAl}$ exhibits a sample-dependent low-temperature transition which may be residual ferromagnetism ${ }^{10,21}$ or a superparamagnetic freezing temperature. ${ }^{7,19}$ Furthermore in $\mathrm{Fe}_{2} \mathrm{VAl}$ and $\mathrm{Fe}_{2} \mathrm{VGa}$ localized magnetic defects have been observed and associated with $\mathrm{Fe}$ antisites, ${ }^{6,12,15}$ while there is also evidence for larger magnetic clusters in the materials. ${ }^{7,19}$ No magnetic splitting was observed in Mössbauer studies near the $\mathrm{Fe}_{2} \mathrm{VAl}$ composition, ${ }^{10,22,23}$ while the dominance of defects and clusters makes a percolative behavior seem likely.

In order to better understand the local magnetic properties and their changes with composition, we have undertaken NMR and Mössbauer shift studies, providing information about on-site electron densities and the local magnetic configurations of $\mathrm{Fe}_{2} \mathrm{VAl}, \mathrm{Fe}_{2} \mathrm{VGa}$, and the mixed composition $\mathrm{Fe}_{2+x} \mathrm{~V}_{1-x} \mathrm{Al}$. In previous NMR studies reported by two of us, ${ }^{16,24}$ the lineshapes were shown to be sensitive probes of the magnetic defects in $\mathrm{Fe}_{2} \mathrm{VAl}$ and $\mathrm{Fe}_{2} \mathrm{VGa}$, while ${ }^{51} \mathrm{~V}$ Korringa relaxation was used to characterize the electronic structure in the region of the semimetallic gap. Here we report measurements of non-Korringa ${ }^{27} \mathrm{Al}$ relaxation behavior near $T_{c}$ in $\mathrm{Fe}_{2+x} \mathrm{~V}_{1-x} \mathrm{Al}$, for compositions bracketing the $x$ $=0$ phase. We show that relaxation peaks near $T_{c}$, together with the lineshapes for these compositions, give a consistent picture of the behavior of antisite defects in this material. The relaxation peaks are due to the critical slowing down of these magnetic defects, and we show that the magnetic defect density tracks the $\mathrm{Fe}$ content for the Fe-rich composition. Furthermore, in ${ }^{57} \mathrm{Fe}$ Mössbauer results we identify the isomer shifts for $8 c$ sites in $\mathrm{Fe}_{2} \mathrm{VAl}$ and $\mathrm{Fe}_{2} \mathrm{VGa}$ to differ very little from those of the corresponding $\mathrm{Fe}$ sites in $\mathrm{Fe}_{3} \mathrm{Al}$ and metastable $\mathrm{Fe}_{3} \mathrm{Ga}$. Thus while the $4 b$-site $\mathrm{Fe}$ content changes, the local electronic structure is relatively little affected by the composition change.

\section{EXPERIMENT}

Samples used for all measurements were a portion of the same ingots used in previous NMR (Refs. 7,16,24) and specific heat ${ }^{6}$ measurements. These are polycrystalline samples prepared by arc melting. Ingots were annealed in vacuum at 800 or $1000^{\circ} \mathrm{C}$, then $400^{\circ} \mathrm{C}$, followed by furnace cooling. $\mathrm{X}$-ray powder analysis on all samples showed the expected 


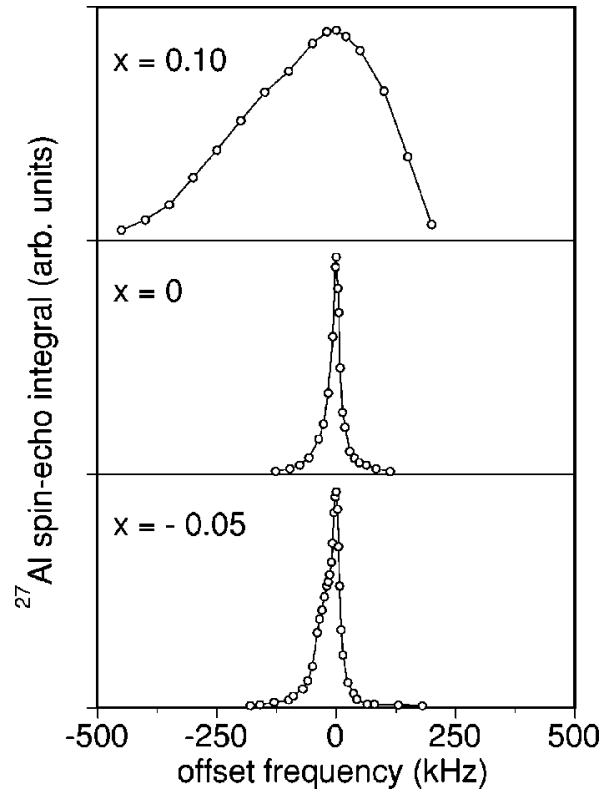

FIG. 1. Room temperature ${ }^{27} \mathrm{Al} \mathrm{NMR}$ powder patterns for $\mathrm{Fe}_{2+x} \mathrm{~V}_{1-x} \mathrm{Al}$ with $x=0.10,0$, and -0.05 , respectively.

$\mathrm{L} 2{ }_{1}$ structure, and microprobe analysis confirmed these to be single-phase. Larger lattice constants were found for the nonstoichiometric $\mathrm{Fe}_{2+x} \mathrm{~V}_{1-x} \mathrm{Al}$ compositions, similar to those reported in the literature. ${ }^{8,9,25}$

NMR experiments were performed at fixed field using a 9-T homebuilt pulse NMR spectrometer described elsewhere. ${ }^{16}{ }^{27} \mathrm{Al}$ NMR spectra for all samples were detected near $99 \mathrm{MHz}$. Ingots were powdered to an approximate 100-200 $\mu \mathrm{m}$ particle size. These powders mixed with granular quartz were placed in thin walled plastic vials for 4-300 K measurements, and Teflon tubes for high temperature purposes. Both sample holders show no observable ${ }^{27} \mathrm{Al}$ NMR signals.

${ }^{57} \mathrm{Fe}$ Mössbauer measurements were obtained using a ${ }^{57}$ Co source in Pd matrix, at ambient temperature, driven in the triangle mode. Results were least-squares fitted using Voigt lineshapes, with shifts referenced to $\alpha$-Fe. The instrumental broadening was small, as estimated from a Sodium Nitroprusside calibration sample; the reported linewidths were corrected for this term.

\section{RESULTS}

${ }^{27} \mathrm{Al}$ room-temperature NMR powder patterns for the $\mathrm{Fe}_{2+x} \mathrm{~V}_{1-x} \mathrm{Al}$ compounds are shown in Fig. 1, measured by spin-echo integration versus frequency. We found relatively narrow NMR linewidths for $\mathrm{Fe}_{2} \mathrm{VAl}$ and $\mathrm{Fe}_{1.95} \mathrm{~V}_{1.05} \mathrm{Al}$, although the latter exhibits an anisotropic line, presumably due to different neighbor configurations in the mixed alloy. The $\mathrm{Fe}_{2.1} \mathrm{~V}_{0.9} \mathrm{Al}$ compound, however, shows a significantly broadened ${ }^{27} \mathrm{Al}$ spectrum, which can be attributed to strong local magnetism, corresponding to the bulk magnetism observed for $x>0 .{ }^{8,9}$

The $4 a \mathrm{Al}$ sites in $\mathrm{Fe}_{2} \mathrm{VAl}$ have cubic site symmetry, and hence no quadrupole broadening for ${ }^{27} \mathrm{Al}$ NMR in the ideal

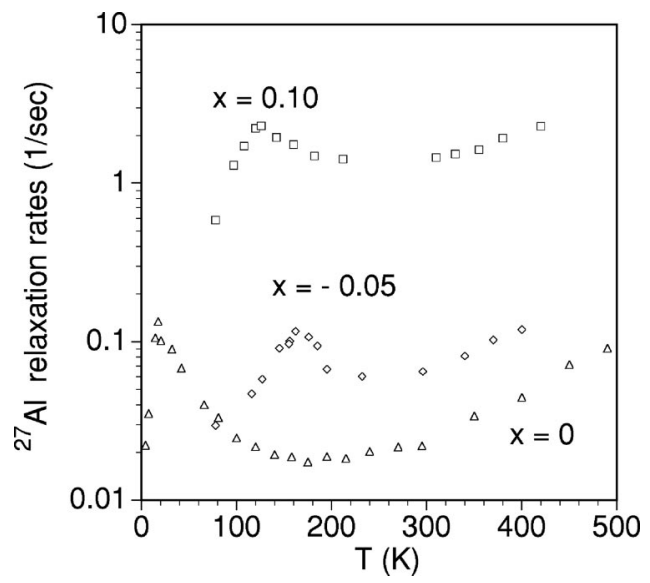

FIG. 2. Temperature dependence of ${ }^{27} \mathrm{Al}$ relaxation rates in $\mathrm{Fe}_{2+x} \mathrm{~V}_{1-x} \mathrm{Al}$, indicated by triangles, circles, and diamonds for $x$ $=0.10,0$, and -0.05 , respectively.

case. However, from pulse-angle studies we determined that the satellite transitions are broadened and made unobservable by random defects, leaving a central line only $(1 / 2 \leftrightarrow-1 / 2$ transition). This is typical behavior for dilute alloys ${ }^{26}$ even for the small defect densities found in stoichiometric $\mathrm{Fe}_{2}$ VAl. In a previous NMR study of $\mathrm{Fe}_{2} \mathrm{VAl}$ (same ingot as the present $x=0$ sample), ${ }^{27} \mathrm{Al}$ and ${ }^{51} \mathrm{~V}$ linewidths were found to be identical, and to have a Curie-law temperature dependence with a limiting high-temperature intrinsic width of $3 \mathrm{kHz}$. The temperature-dependence is characteristic of broadening by local moments, and was shown to be consistent with the presence of magnetic antisite defects. ${ }^{7}$ Clearly for the $x \neq 0$ cases the larger linewidths (Fig. 1) must be caused by inhomogeneous broadening due to the larger defect density in these alloys. From limited temperaturedependence studies we observed the nonstoichiometric samples to have linewidths increasing with decreasing temperature, as would be expected. We measured the spin-echo $T_{2}$ and observed a weak temperature increase at low temperatures, to a value of $210 \mu \mathrm{s}$ at $4 \mathrm{~K}$ for $x=0$, consistent with the freezing-in of local fields as the system goes through its spin freezing at low temperatures. ${ }^{7}$ Maksimov et al. ${ }^{10}$ have similarly seen a suppression of dynamic relaxation processes at the spin freezing point, in a $\mu$ SR study.

The antisite-defect dynamics are manifested much more clearly in the ${ }^{27} \mathrm{Al} T_{1}$, rather than the $T_{2}$, and this provides a powerful tool to investigate the spin dynamics. The ${ }^{51} \mathrm{~V} T_{1}$ exhibits Korringa behavior at low temperatures, indicating that conduction electrons dominate the relaxation of this nucleus. However, the ${ }^{27} \mathrm{Al} T_{1}$ was previously noted to exhibit non-Korringa relaxation in $\mathrm{Fe}_{2} \mathrm{VAl}{ }^{16,24}$ Here we report results of a detailed study showing a clear $T_{1}^{-1}$ peak for $\mathrm{Fe}_{2} \mathrm{VAl}$, and similar behavior in the $x \neq 0$ materials. Results are shown in Fig. 2 by squares, triangles, and diamonds, for $x=0.10,0$, and -0.05 , respectively. Rates were measured by inversion recovery, using the integral of the spin echo fast Fourier transform, irradiating the central portion of the ${ }^{27} \mathrm{Al}$ line. For the recovery of the central transition, the $T_{1}$ 's were extracted by fitting to multiexponential curves ${ }^{27}$ appropriate for magnetic relaxation of the $I=5 / 2{ }^{27} \mathrm{Al}$ central transition. 


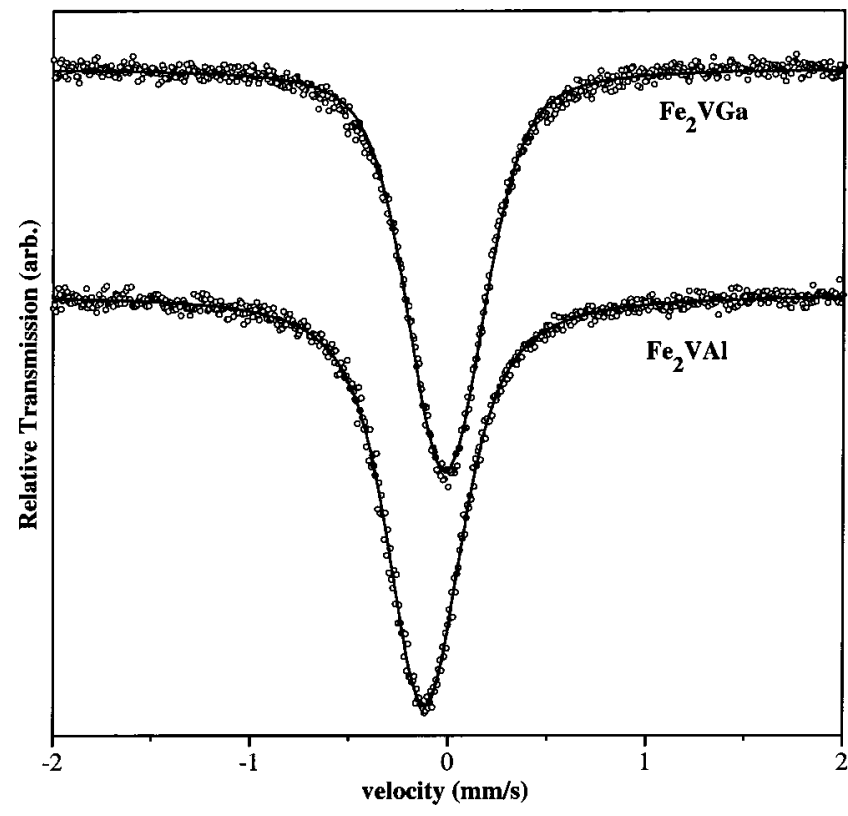

FIG. 3. Room-temperature Mössbauer spectra for $\mathrm{Fe}_{2} \mathrm{VAl}$ and $\mathrm{Fe}_{2} \mathrm{VGa}$. Least-squares fits are superposed on the data. Velocities are relative to the source.

$T_{1}^{-1}$ maxima indicate the critical slowing down of the local field fluctuations at the nuclear sites. ${ }^{28,29} \mathrm{We}$ find that ${ }^{27}\left(T_{1}^{-1}\right)$ passes through a maximum at $125 \mathrm{~K}, 17 \mathrm{~K}$, and 165 $\mathrm{K}$ for $\mathrm{Fe}_{2.1} \mathrm{~V}_{0.9} \mathrm{Al}, \mathrm{Fe}_{2} \mathrm{VAl}$, and $\mathrm{Fe}_{1.95} \mathrm{~V}_{1.05} \mathrm{Al}$, respectively. On further cooling, ${ }^{27}\left(T_{1}^{-1}\right)$ drops rapidly in all samples. These peaks correspond to susceptibility peaks observed in the same samples. ${ }^{7}$ For $\mathrm{Fe}_{2.1} \mathrm{~V}_{0.9} \mathrm{Al}$ this peak corresponds to a ferromagnetic ordering temperature, while for $\mathrm{Fe}_{2} \mathrm{VAl}$ the transition may be a superparamagnetic freezing temperature. ${ }^{7}$ It is not entirely clear why no peak was observed for ${ }^{51}\left(T_{1}^{-1}\right)$, although for the latter, the presence of $d$ orbitals significantly enhances the Korringa relaxation process, while a small RKKY term might also contribute and be important for ${ }^{51} \mathrm{~V}$.

Room-temperature Mössbauer measurements for both $\mathrm{Fe}_{2} \mathrm{VAl}$ and $\mathrm{Fe}_{2} \mathrm{VGa}$ yielded single lines, as shown in Fig. 3. The data are shown in the figure, along with the least-squares fitting curves. In both cases, a good fit was obtained using a single Voigt-broadened line. The two linewidths thus determined are identical, and slightly larger than the natural width for Fe. Parameters from these fits are given in Table I. The widths given in the table are excess values over the natural width for Fe. These small widths indicate a relative lack of inhomogeneous broadening, showing these samples to be well-ordered in the Heusler structure, with very similar Fe site occupancies. Also shown in the table are isomer shifts for $\mathrm{Fe}_{3} \mathrm{Al}$ and $\mathrm{Fe}_{3} \mathrm{Ga}$, from Lin et al. ${ }^{30}$ and Kawamiya et al. ${ }^{31}$ respectively. There have been a number of Mössbauer studies of the stable compound $\mathrm{Fe}_{3} \mathrm{Al},{ }^{20,30,32,33}$ while for $\mathrm{Fe}_{3} \mathrm{Ga}$ the $\mathrm{DO}_{3}$ structure is metastable, and quenched samples were used to obtain the referenced values. Finally, for comparison, values for the vanadium Knight shift and the cubic lattice constant of $\mathrm{Fe}_{2} \mathrm{VAl}$ and $\mathrm{Fe}_{2} \mathrm{VGa}$ are shown in
TABLE I. Measured Mössbauer isomer shifts and widths, along with NMR Knight shifts and lattice parameters. Room-temperature isomer shifts $(\delta)$ and line widths $(\sigma)$ are listed. Isomer shifts are quoted relative to an $\alpha$-Fe reference. Vanadium Knight shifts $\left(K_{V}\right)$ are low-temperature values. Wyckoff positions labels are given for the $\mathrm{DO}_{3}$ lattice (equivalent to the $\mathrm{L} 21$ labels).

\begin{tabular}{llllll}
\hline \hline Material & $a(\mathrm{~nm})$ & Site & $\delta(\mathrm{mm} / \mathrm{s})$ & $\sigma(\mathrm{mm} / \mathrm{s})$ & $K_{V}(\%)$ \\
\hline $\mathrm{Fe}_{2} \mathrm{VAl}$ & $0.576^{\mathrm{b}}$ & $8 c$ & $0.058(5)^{\mathrm{a}}$ & $0.054(5)^{\mathrm{a}}$ & $0.61^{\mathrm{b}}$ \\
$\mathrm{Fe}_{3} \mathrm{Al}$ & & $8 c$ & $0.05^{\mathrm{c}}$ & & \\
& & $4 b$ & $0.19^{\mathrm{c}}$ & & \\
$\mathrm{Fe}_{2} \mathrm{VGa}$ & $0.577^{\mathrm{b}}$ & $8 c$ & $0.161(5)^{\mathrm{a}}$ & $0.054(5)^{\mathrm{a}}$ & $0.59^{\mathrm{b}}$ \\
$\mathrm{Fe}_{3} \mathrm{Ga}$ & & $8 c$ & $0.18^{\mathrm{d}}$ & & \\
& & $4 b$ & $0.28^{\mathrm{d}}$ & & \\
\hline \hline
\end{tabular}

${ }^{\mathrm{a}}$ This work.

${ }^{\mathrm{b}}$ Reference 7 .

${ }^{\mathrm{c}}$ Reference 30 .

${ }^{\mathrm{d}}$ Reference 31 .

the table. These values are quite close, indicating the similarity of these two materials.

\section{DISCUSSION}

Previously, the temperature dependences of the ${ }^{27} \mathrm{Al}$ and ${ }^{51} \mathrm{~V}$ linewidths in $\mathrm{Fe}_{2} \mathrm{VAl}$ were fit using the analytical results of Walstedt and Walker ${ }^{34}$ for dipolar broadening due to random local-moment spins. Taking the local moments to be antisite defects of $3.7 \mu_{B}$ as obtained from specific heat measurements, ${ }^{6}$ this fit yielded a defect concentration of $c$ $=0.0045,{ }^{7}$ per formula unit, which compares favorably with the value $c=0.0037$ obtained from specific heat. In the dilute limit, the line shape for this mechanism will be Lorentzian, with identical widths for ${ }^{27} \mathrm{Al}$ and ${ }^{51} \mathrm{~V}$, as indeed observed in $\mathrm{Fe}_{2} \mathrm{VAl}^{7}{ }^{7}$ These results gives confidence that the broadening mechanism and the defect concentration are well understood. Note that several groups have identified the presence of large-moment clusters in addition to the antisite local moments in $\mathrm{Fe}_{2} \mathrm{VAl}^{7,19}$ however these turn out to have a much smaller contribution to the $\mathrm{Fe}_{2} \mathrm{VAl}$ NMR linewidth. ${ }^{7}$

For dipolar broadening in NMR, in the dilute limit one finds that the Lorentzian line has a width proportional to $c .^{34}$ From the data of Fig. 1 we find that the $x=0.10$ sample exhibits a half-width 17 times larger than that of the $x=0$ sample. Nominally this implies an antisite concentration for $x=0.10$ of $c=0.077$ per formula unit, and although this value is outside the dilute limit where the analytic expressions are no longer exact, this provides an approximate estimate of the density of local defects. This sample contains $c$ $=0.10$ excess $\mathrm{Fe}$ per formula unit, so the model described here shows that a sizable portion (possibly all) of the excess $\mathrm{Fe}$ is distributed as random defects on $\mathrm{V}$ sites, rather than clustered within the sample.

NMR spin-lattice relaxation due to an uncorrelated population of local moments has been treated by several authors. ${ }^{29,35-38}$ If nuclear spin-diffusion is not important, the relaxation function is a stretched exponential, $s(t)$ 
$\propto \exp -\left(t / \tau_{1}\right)^{1 / 2}$, due to an inhomogeneous distribution of local relaxation rates. For a concentration $n$ per unit volume of effective moments $p$, the exponential factor is, ${ }^{36}$

$$
\tau_{1}^{-1}=0.84 \frac{p \mu_{B} \gamma_{n} n}{\sqrt{\omega}}\left(\frac{\omega \tau_{c}}{1+\omega^{2} \tau_{c}^{2}}\right)^{1 / 2},
$$

where $\omega$ is the NMR frequency, and a single Debye-type correlation time $\tau_{c}$ has been assumed to apply to the local moments. At a temperature where $\tau_{c}=\omega$, a maximum occurs in Eq. (1), giving $\tau_{1}^{-1}=0.19 \mathrm{~s}^{-1}$ for our $x=0$ sample, using the values $p=3.7$ and $n=7.6 \times 10^{19} \mathrm{~cm}^{-3}$ (corresponding to $c=0.0037)$, values taken from the specific heat measurement ${ }^{7}$ and described above. A distribution of correlation times will give a somewhat lower peak value. In our $T_{1}^{-1}$ measurements, for each temperature the signal amplitude was measured over one or two decades of recovery time, and a best fit was made to the three-exponential $I$ $=5 / 2$ magnetic relaxation function for central transition inversion-recovery. ${ }^{27}$ For central-transition measurements, the stretched-exponential curve for local-moment relaxation should be convoluted with the same $I=5 / 2$ multiexponential. From numerical plots we found that such a convolution is nearly indistinguishable from the three-exponential recovery curve if $\tau_{1}=T_{1}$ over about one decade of recovery time. The peak value observed for $x=0$ (Fig. 2) is $T_{1}^{-1}=0.13 \mathrm{~s}^{-1}$, in good agreement with the value $0.19 \mathrm{~s}^{-1}$ obtained from the model described here. Therefore, just as for the line shapes, the density of local moments obtained independently, and attributed to antisite defects, is in good quantitative agreement with the observed ${ }^{27} T_{1}^{-1}$ peak value in the $x=0$ sample.

Local-moment relaxation of this type scales with $n$ [Eq. (1), exact in the limit of dilute moments]. From the data in Fig. 2, the peak value of $T_{1}^{-1}$ is found to be 17 times larger for the $x=0.10$ sample than for the $x=0$ sample. This is the same ratio as found for the linewidths, thus giving quantitative consistency with the model, showing that the excess Fe act as local moments fluctuating independently in the paramagnetic regime at this concentration. The peaks observed in $T_{1}^{-1}$ coincide with maxima in the ac susceptibility, ${ }^{39}$ and therefore correspond to magnetic transitions in the samples. The relaxation peaks can be attributed to critical slowing down of the local moment dynamics at these transition temperatures. Maxima in the $\mu \mathrm{SR}$ longitudinal relaxation rate were also observed by Maksimov, et al. ${ }^{10}$ attributed to the same mechanism.

For the $x=-0.05$ sample the density of local moments is smaller than for the $x=0.10$ sample; this can be seen from both the NMR linewidth and the peak value of ${ }^{27} T_{1}^{-1}$. On the other hand, the transition temperature for this sample is the largest of the three. Of course, the local moment density might be expected to be smaller since there is no Fe excess in this sample. Thus, the moment density does not necessarily correlate with $T_{c}$ in this material. Indeed, as has been pointed out previously, ${ }^{10}$ the local moment density is below the per- colation limit in all of these samples, and thus it is not sensible that the magnetic transitions are driven solely by the interaction of these moments.

Another feature identified in $\mathrm{Fe}_{2} \mathrm{VAl}$ is the presence of a more dilute set of large moments. ${ }^{7,19}$ These were shown previously to induce superparamagnetic behavior in $\mathrm{Fe}_{2} \mathrm{VAl},{ }^{7}$ although their contribution to the NMR linewidth is small. The spin-lattice relaxation time will also not be affected by these moments, since they will be saturated in the measuring magnetic field. Since no second phase was observed in electron microprobe measurements, we propose that the large moments are ferromagnetic regions in the Heusler lattice having enhanced conduction electron density, perhaps associated with variations in $\mathrm{Fe}$ concentration. Indeed, $\mathrm{Fe}_{2} \mathrm{VAl}$ is a semimetal, but with increasing $x$ becomes ferromagnetic, its metallic bands becoming occupied. ${ }^{9,24}$ The interaction of locally ferromagnetic regions can lead to superparamagnetic freezing in $\mathrm{Fe}_{2} \mathrm{VAl}$, while with increasing $x$ these regions percolate, leading to ferromagnetic ordering. According to

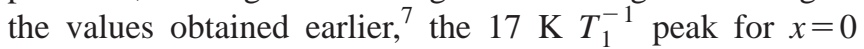
must be due to field polarization of the antisite spins, while the higher-temperature peaks for $x \neq 0$ must be due to antisite defects which are coupled by band electrons.

With sufficient $\mathrm{Fe}$ concentration $\mathrm{Fe}_{2+x} \mathrm{~V}_{1-x} \mathrm{Al}$ will approach $\mathrm{Fe}_{3} \mathrm{Al}$, in which the $8 c$ sites carry a moment, in addition to the $4 b$ sites (antisites for $\mathrm{Fe}_{2} \mathrm{VAl}$ ). ${ }^{20}$ Mössbauer measurements give us a measure of the electronic structure changes involved in this behavior. We have found (Table I) that the isomer shift for $\mathrm{Fe}_{2} \mathrm{VAl}$ is nearly identical to that of the corresponding $8 c$ site in $\mathrm{Fe}_{3} \mathrm{Al}$. A similar result is seen for $\mathrm{Fe}_{2} \mathrm{VGa}$ and $\mathrm{Fe}_{3} \mathrm{Ga}$. A large change in average isomer shift was found vs V substitution by Shobaki et al., ${ }^{23}$ but this can be attributed to a change in Fe site occupation. Since the most important contribution to the isomer shift is due to the on-site $s$-electron concentration, clearly very little electron transfer into $s$-orbits is involved in the substitution of Fe for V. We conclude that at least in this regard, the Fe local electronic structure is not substantially changed by the change of its neighbors from $\mathrm{V}$ to $\mathrm{Fe}$. We picture the addition of $\mathrm{Fe}$ leading to the filling of $d$-bands in a manner rather like that of a rigid band model.

The isomer shift for $\mathrm{Fe}_{3} \mathrm{Ga}$ is significantly different from that of $\mathrm{Fe}_{3} \mathrm{Al}$. This seems surprising given the close similarity of the two materials. For example, the vanadium Knight shifts quoted in Table I are nearly identical, and these measure the orbital susceptibility of the $d$-bands in the two materials. However, large isomer shifts have also been observed in $\mathrm{Fe}_{3} \mathrm{Ga}_{4} \cdot{ }^{40}$ Apparently the larger Ga atom leads to more covalent bonding, thus stabilizing $p$ and $d$ orbitals relative to $s$.

\section{CONCLUSIONS}

A measurement of the ${ }^{27} \mathrm{Al}$ NMR line shapes and spinlattice relaxation in $\mathrm{Fe}_{2+x} \mathrm{~V}_{1-x} \mathrm{Al}$ showed that a consistent measure of the local moment density and dynamics could be obtained. For $x=0.10$, the local moment density is approximately equal to the excess Fe concentration. This density is below the percolation limit, although the material has a fer- 
romagnetic transition. The development of a metallic band can explain this transition. Mössbauer measurements show that little change in Fe local electronic structure accompanies the change from semimetallic $\mathrm{Fe}_{2} \mathrm{VAl}$ to ferromagnetic $\mathrm{Fe}_{3} \mathrm{Al}$, with a similar result found for $\mathrm{Fe}_{2} \mathrm{VGa}$.

\section{ACKNOWLEDGMENTS}

This work was supported by the Robert A. Welch Foundation, Grant No. A-1526, and the National Science Foundation (DMR-0103455).
${ }^{1}$ A.E. Berkowitz, J.R. Mitchell, M.J. Carey, A.P. Young, S. Zhang, F.E. Spada, F.T. Parker, A. Hutten, and G. Thomas, Phys. Rev. Lett. 68, 3745 (1992).

${ }^{2}$ J.Q. Xiao, J.S. Jiang, and C.L. Chien, Phys. Rev. Lett. 68, 3749 (1992).

${ }^{3}$ J.M.D. Coey, in Aspects of Modern Magnetism, edited by F.C. Pu, Y.J. Wang, and C.H. Shang (World Scientific, Singapore, 1996), p. 2.

${ }^{4}$ K. Endo, H. Matsuda, K. Ooiwa, M. Iijima, T. Goto, K. Sato, and I. Umehara, J. Magn. Magn. Mater. 177-181, 1437 (1998).

${ }^{5}$ H. Matsuda, K. Endo, K. Ooiwa, M. Iijima, Y. Takano, H. Mitamura, T. Goto, M. Tokiyama, and J. Arai, J. Phys. Soc. Jpn. 69, 1004 (2000).

${ }^{6}$ C.S. Lue, J.H. Ross, Jr., C.F. Chang, and H.D. Yang, Phys. Rev. B 60, R13 941 (1999)

${ }^{7}$ C.S. Lue, J.H. Ross, Jr., K.D.D. Rathnayaka, D.G. Naugle, S.Y. Wu, and W.-H. Li, J. Phys.: Condens. Matter 13, 1585 (2001).

${ }^{8}$ Y. Nishino, M. Kato, S. Asano, K. Soda, M. Hayasaki, and U. Mizutani, Phys. Rev. Lett. 79, 1909 (1997).

${ }^{9}$ M. Kato, Y. Nishino, U. Mizutani, and S. Asano, J. Phys.: Condens. Matter 12, 1769 (2000).

${ }^{10}$ I. Maksimov, D. Baabe, H.H. Klauss, F.J. Litterst, R. Feyerherm, D.M. Többens, A. Matsushita, and S. Süllow, J. Phys.: Condens. Matter 13, 5487 (2001).

${ }^{11}$ G.Y. Guo, G.A. Botton, and Y. Nishino, J. Phys.: Condens. Matter 10, L119 (1998).

${ }^{12}$ D.J. Singh and I.I. Mazin, Phys. Rev. B 57, 14352 (1998).

${ }^{13}$ R. Weht and W.E. Pickett, Phys. Rev. B 58, 6855 (1998).

${ }^{14}$ M. Weinert and R.E. Watson, Phys. Rev. B 58, 9732 (1998).

${ }^{15}$ A. Bansil, S. Kaprzyk, P.E. Mijnarends, and J. Tobola, Phys. Rev. B 60, 13396 (1999).

${ }^{16}$ C.S. Lue and J.H. Ross, Jr., Phys. Rev. B 58, 9763 (1998).

${ }^{17}$ In the previous reference, labels on the vertical axis of Fig. 3(a) were a factor of 10 too small. This does not affect the conclusions of that paper.

${ }^{18}$ H. Okamura et al., Phys. Rev. Lett. 84, 3674 (2000).
${ }^{19}$ Y. Feng, J.Y. Rhee, T.A. Wiener, D.W. Lynch, B.E. Hubbard, A.J. Sievers, D.L. Schlagel, T.A. Lograsso, and L.L. Miller, Phys. Rev. B 63, 165109 (2001).

${ }^{20}$ M.B. Stearns, Phys. Rev. 168, 588 (1968).

${ }^{21}$ A. Matsushita and Y. Yamada, J. Magn. Magn. Mater. 196-197, 669 (1999).

${ }^{22}$ E. Popiel, M. Tuszynski, W. Zarek, and T. Rendecki, J. LessCommon Met. 146, 127 (1989).

${ }^{23}$ J. Shobaki, I.A. Al-Omari, M.K. Hasan, K.A. Azez, M.-A.H. AlAkhras, B.A. Albiss, H.H. Hamdeh, and S.H. Mahmood, J. Magn. Magn. Mater. 213, 51 (2000).

${ }^{24}$ C.S. Lue and J.H. Ross, Jr., Phys. Rev. B 61, 9863 (2000).

${ }^{25}$ W. Zarek, E. Talik, J. Heimann, M. Kulpa, A. Winiarska, and M. Neumann, J. Alloys Compd. 297, 53 (2000).

${ }^{26} \mathrm{O}$. Kanert and M. Mehring, in NMR: Basic Principles and Progress, edited by E. Fluck and R. Kosfeld (Springer-Verlag, New York, 1971), Vol. 3, p. 1.

${ }^{27}$ A. Narath, Phys. Rev. 162, 320 (1967).

${ }^{28}$ T. Moriya, Spin Fluctuations in Itinerant Electron Magnetism (Springer-Verlag, Berlin, 1985).

${ }^{29}$ A. Narath, CRC Crit. Rev. Solid State Sci. 3, 1 (1972).

${ }^{30}$ M.-C. Lin, R.G. Barnes, and D.R. Torgeson, Phys. Rev. B 24, 3712 (1981).

${ }^{31}$ N. Kawamiya, K. Adachi, and Y. Nakamura, J. Phys. Soc. Jpn. 33, 1318 (1972).

${ }^{32}$ S.B. Raju, J.P. Eymery, and P. Moine, Scr. Metall. 13, 649 (1979).

${ }^{33}$ B. Fultz, Z. Gao, H.H. Hamdeh, and S.A. Oliver, Phys. Rev. B 49, 6312 (1994).

${ }^{34}$ R.E. Walstedt and L.R. Walker, Phys. Rev. B 9, 4857 (1974).

${ }^{35}$ I.J. Lowe and D. Tse, Phys. Rev. 166, 279 (1968).

${ }^{36}$ D. Tse and S.R. Hartmann, Phys. Rev. Lett. 21, 511 (1968).

${ }^{37}$ C.C. Sung and L.G. Arnold, Phys. Rev. B 7, 2095 (1973).

${ }^{38}$ G.B. Furman, E.M. Kunoff, S.D. Goren, V. Pasquier, and D. Tinet, Phys. Rev. B 52, 10182 (1995).

${ }^{39}$ C.S. Lue, Ph.D. thesis, Texas A\&M University, 1999.

${ }^{40}$ M.A. Kobeissi, J.A. Hutchings, P.G. Appleyard, M.F. Thomas, and J.G. Booth, J. Phys.: Condens. Matter 11, 6251 (1999). 